\title{
Effect of Occipital Nerve Stimulation (ONS) on the Orbicularis Oculi Reflex Triggered by a Standardized Air Flow in Patients with Chronic Migraine-A Prospective, Randomized, Interventional Study
}

Carl H. Göbel (1) · Axel Heinze · Sarah Karstedt · Svenja Clasen •

Hartmut Göbel (10)

Received: January 8, 2021 / Accepted: February 4, 2021 / Published online: February 25, 2021

(c) The Author(s) 2021

\section{ABSTRACT}

Introduction: Occipital nerve stimulation (ONS) is a specific form of peripheral neuromodulation used in the treatment of chronic pain disorders. A particular field of application is in the therapy of treatment-refractory headaches, especially of chronic migraine. The precise mode of action is unknown. It is presumed that central and peripheral sensitization are reduced in patients with chronic headache. The aim of this study was to examine the effect of ONS on pain-modulatory mechanisms in the trigeminocervical area in patients with chronic migraine.

Methods: In a balanced repeated measurements design in eight patients with chronic migraine with and without active ONS, we analyzed

C. H. Göbel $(\bowtie) \cdot$ S. Karstedt

Department of Neurology, University Hospital Schleswig-Holstein, Campus Lübeck, Germany e-mail: carl.goebel@neuro.uni-luebeck.de

C. H. Göbel · A. Heinze · S. Karstedt · S. Clasen ·

H. Göbel

Kiel Migraine and Headache Centre, Kiel, Germany which effects ONS had on the orbicularis oculi reflex dynamically elicited by corneal air flow.

Results: The orbicularis oculi reflex in active ONS $\quad(7.38 \pm 20.14$ eyelid closures/minute) compared to inactive ONS $(18.73 \pm 14.30$ eyelid closures/minute) is significantly reduced $(p=0.021)$.

Conclusions: The results show that under active ONS compared to inactive ONS in patients with chronic migraine, the orbicularis oculi reflex, dynamically triggered by a standardized air flow, is significantly reduced. This suggests that ONS is able to directly counteract the trigeminally mediated central sensitization in chronic migraine and protectively reduce the effects of aversive peripheral stimulation.

Keywords: Blink reflex; Central sensitization; Chronic migraine; Dynamic stimulation; Mode of action; Orbicularis oculi reflex; Occipital nerve stimulation; ONS; Peripheral sensitization; Standardized air flow 


\section{Key Summary Points}

Why carry out this study?

Occipital nerve stimulation (ONS) is a special form of peripheral neuromodulation for the treatment of chronic pain disorders, especially treatment-refractory headaches such as chronic migraines.

The exact mechanism of action is not known. A reduction in central and peripheral sensitization in patients with chronic headaches is assumed.

In order to confirm this hypothesis, patients with chronic migraine with and without active ONS should be investigated to determine which effects the ONS has on the orbicularis-oculi-reflex triggered dynamically with an air stream.

\section{What was learned from the study?}

The results show that in active ONS compared to inactive ONS in patients with chronic migraine, the orbicularis oculi reflex is significantly reduced.

This indicates that ONS is able to directly counteract the trigeminally mediated central sensitization in chronic migraine. This could protectively reduce the effects of aversive peripheral irritation.

\section{DIGITAL FEATURES}

This article is published with digital features, including a summary slide, to facilitate understanding of the article. To view digital features for this article go to https://doi.org/10.6084/ m9.figshare.13713841.

\section{INTRODUCTION}

Occipital nerve stimulation (ONS) is a specific form of peripheral neuromodulation used in the treatment of chronic pain disorders. A particular field of application is in the therapy of treatment-refractory headaches, especially of chronic migraine $[3,5,8,10,12,15,22,24$, $25,27,29,31,37]$. ONS targets central pain processing mechanisms by electrical stimulation of the greater occipital nerve. The idea of using occipital nerve stimulation for the treatment of headache was described as early as 1977 [23]. In 1999, Weiner and Reed published its application in 12 patients suffering from occipital neuralgia [34]. This study was the starting point for a large number of further studies on clinical application and possible mechanisms of action [3, 5, 11, 12, 19, 20, 22, $25,26,29,35]$. Neuromodulation aims to modulate the function of the anatomical structures and pathways that play a role in headache mechanisms for therapeutic purposes. External electrical stimuli are intended to act therapeutically on intrinsic electrical signals and pain modulation [16].

The exact mode of action of the ONS is not yet known. Several theories are discussed. In chronic migraine, increased sensitization of pain circuitry with increased ventral posteromedial nucleus (VPM) neuronal firing is assumed. The mode of action of ONS could involve attenuation of neurons in the VPM of $\mathrm{CM}$ rats [33]. An effect of the ONS on central sensitization and impaired conditioned pain modulation (CPM) was also shown in humans. ONS stimulates the distal branches of $\mathrm{C} 1, \mathrm{C} 2$, and C3. This enables the procedure to possibly alter the nociceptive traffic to the trigeminocervical complex, brainstem, and supranuclear connections. Under ONS, a normalization of the CPM response following ONS was shown. This could indicate a reduction in central sensitization through ONS in migraine patients [36]. ONS modulates the responses of the 'trigeminocervical complex' (TCC) neurons to light mechanical input by GABAergic and glycinergic mechanisms. This modulation is mediated by GABAergic and glycinergic 
mechanisms and can have an inhibitory effect on nociception in the spinal and medullary dorsal horn neurons [9]. In a positron emission tomography (PET) study, ONS stimulation resulted in activation in the dorsal lateral prefrontal cortex, comprising the medial pain pathway, the ventral medial prefrontal cortex, and the bilateral anterior cingulate cortex as well as the parahippocampal area. The latter two areas form the descending pain pathway. In addition, a relative deactivation was observed in the left somatosensory cortex, constituting the lateral pain pathway as well as other sensory areas. The results suggest increased activity in the descending pain pathway [1].

The studies mentioned above indicate a reduction in central and peripheral sensitization in patients with chronic headaches. A particularly sensitive protective reflex in the trigeminal area is the orbicularis oculi reflex. The orbicularis oculi reflex (also lid closure reflex, corneal reflex, blink reflex) is a reflective antinociceptive protective mechanism of the eye to ward off damage $[7,14,18,21,28]$. It can be triggered by stimulating the cornea or the periorbital area. The reflex response is a rapid closing of the eyelids. The protective reflex fends off foreign bodies, dehydration, and damage to the eyeball. It can also involuntarily occur in the event of light irritation, sudden acoustic stimuli, or emotional reactions such as startling. It can be modulated by centrally acting analgesics [6].

The afferent reflex arc of the orbicularis oculi reflex runs via the ophthalmic branch of the trigeminal nerve in the case of tactile stimuli, and via the optic nerve in the case of optical stimuli. After transmission in the trigeminal complex, the signal is conducted via the superior colliculus or the red nucleus to the reticular formation. From there, it reaches the facial nerve nucleus in the brainstem and causes a contraction of the orbicularis oculi muscle via the efferent arc, the facial nerve [18, 21, 30, 32]. The static electrical stimulus used in routine clinical examination corresponds only remotely to the natural function of the reflex. We therefore opted for using a standardized dynamic air flow corresponding to a more physiological setting. This also allows monitoring the reflex activity continuously over a defined period of time. In contrast to the statically triggered orbicularis oculi reflex by electrical stimulation, the triggering of the reflex response by a permanent air flow represents a dynamic reproduction of the natural reflex situation. This can possibly map endogenous pain-modulating processes in the central nervous system by summing them over time and predict therapeutic processes in a more relevant fashion $[1,9,13]$.

To measure the effect of ONS on the orbicularis oculi reflex triggered as naturally as possible, a new quantitative method was developed for standardized measurement of the orbicularis oculi reflex triggered via an air stream. To stimulate the reflex response, instead of an artificial electrical stimulus, a standardized air flow is directed onto the cornea of the eye. The reflex response is recorded using a video camera detecting eyelid closure frequency (documented as eyelid closures per minute). This method aims to measure the anti-nociceptive protective mechanism of the orbicularis oculi reflex in a way as physiological as possible. At the same time, it allows recording the reflex response dynamically averaged over a longer period of time. The aim of this study was to use this new method in patients with chronic migraine to investigate whether ONS has a direct effect on pain-modulating mechanisms in the trigeminocervical area.

\section{METHODS}

\section{Study Design and Ethics Statement}

In a randomized balanced repeated measures within-subject design, patients were assessed that had previously received ONS therapy due to treatment-refractory chronic migraine. The ethics committee of the medical faculty of the Christian-Albrechts University approved the study (D426/14). All subjects gave their informed written consent prior to participation. The study was performed in agreement with the Declaration of Helsinki.

Inclusion criteria were the diagnosis of chronic migraine as underlying disease and 
treatment with occipital nerve stimulation. Exclusion criteria were scalp injury, severe psychological comorbidities, pregnancy, and/or lack of consent to participate in the study.

The study was divided into two parts, the ON phase with active ONS, and the OFF phase with inactive ONS. In the former, the orbicularis oculi reflex was recorded quantitatively with active ONS. The OFF phase included the measurement of the orbicularis oculi reflex with ONS deactivated. There was a $1-\mathrm{h}$ break between the two test runs. In order to rule out a sequence effect, the patients were randomized into two groups: One group (A) first went through the ON-phase measurement and, after an hour's break, the OFF-phase measurement. In the second group (B), the OFF-phase measurement was started and the ON-phase measurement was carried out $1 \mathrm{~h}$ later.

Group A came to the study appointment with active stimulation and the ON-phase measurement took place first. After completion, the ONS device was switched off. The OFF-phase measurement was made after $1 \mathrm{~h}$. Group B patients switched off the ONS $12 \mathrm{~h}$ prior to the

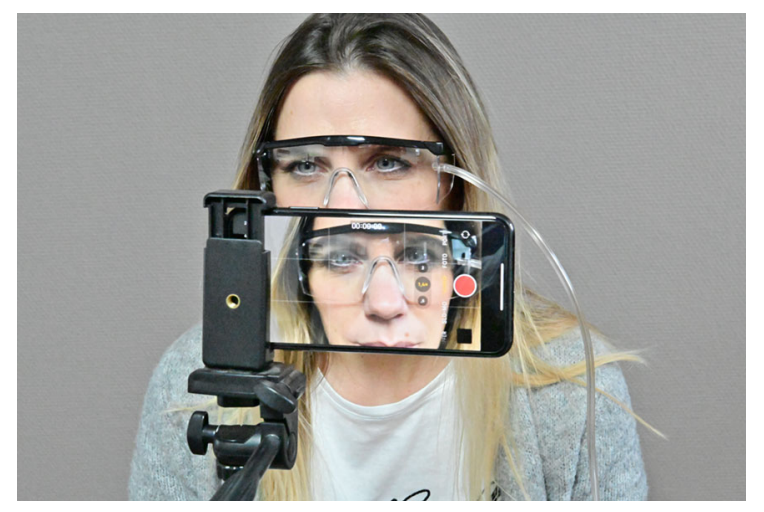

Fig. 1 Standardized quantitative measurement of the orbicularis oculi reflex triggered dynamically with an air stream. The patients wear protective glasses. On the left side, there is a circular opening ( $7 \mathrm{~mm}$ diameter). An air hose is inserted into this side opening of the glasses. This is connected to an oxygen bottle, from which oxygen flows constantly at $7 \mathrm{l} / \mathrm{min}$. The air flow served as a standardized stimulus for the activation of the orbicularis oculi reflex. To quantitatively record the blink frequency as a reflex response, the eyelid closures were filmed with a video camera through the protective glasses and documented in a standardized way (eyelid closures per minute) measurements. The OFF measurements were initially carried out in these participants. Then the ONS was activated and after $1 \mathrm{~h}$ the measurements were repeated with the stimulation switched on (ON phase).

\section{Orbicularis Oculi Reflex Triggered by a Standardized Air Flow}

A new quantitative method was developed for the standardized measurement of the orbicularis oculi reflex. The patient received protective transparent glasses with a circular opening $0.7 \mathrm{~cm}$ wide on the left side (Fig. 1). An air hose was inserted into this opening. This was connected to an oxygen bottle from which oxygen was flowing at a constant rate of $7 \mathrm{l} / \mathrm{min}$. This air flow served as a standardized stimulus for triggering the reflex. To quantitatively record the reflex response, the blinking of the eyes through the protective glasses was filmed with a video camera and documented in a standardized manner. The air flow was active for $1 \mathrm{~m}$. During this time, the blink frequency was recorded contactlessly with the camera. The measurements without airflow (baseline) and with airflow were carried out every 5 min in order to rule out possible habituation effects. The number of times the patient blinked per minute under the respective conditions (spontaneous or with experimental stimulation by the air flow) was counted as the dependent variable. Primary endpoint was the difference in the frequency of lid closure with experimental stimulation and without experimental stimulation with active or inactive ONS.

\section{Surgical Technique}

The peripheral nerve stimulation systems used for occipital nerve stimulation for the treatment of chronic, intractable migraine consisted of an implantable pulse generator (IPG), which produces therapeutic electrical stimulation pulses. All subjects underwent implantation of the ONS device Eonmini ${ }^{\mathrm{TM}}$ (St. Jude Medical Inc., Little Canada, MN, USA). Two leads deliver the therapeutic stimulation pulses to the target location, each using eight electrode poles with 
$4 \mathrm{~mm}$ distance in between. An external handheld device was used to adjust the intensity for each electrode pole for the stimulation. The leads were percutaneously implanted through a needle under general anesthesia. Once the lead was positioned in the desired location, it was anchored to the surrounding tissue to provide stability and avoid migration. Surgical implantation was carried out by two neurosurgical centers by two neurosurgeons. One center performed a trial period of 3 days after implantation to evaluate electrode positioning during this period. The other center implanted electrodes and IPG in one setting without a trial period. In this procedure, a pocket was created in the desired location in which the permanent IPG was stored. The IPG was implanted at the same anatomical location in both centers. Once the pocket was created, a subcutaneous path was created using a tunneling tool to allow the lead to be connected to the IPG. Once the lead was connected to the IPG and the system tested, the pocket was sutured and bandaged. After the permanent IPG was implanted, an external handheld device was used for programming. These devices were used by the clinician to select the stimulation parameters and by the patient to adjust the therapy levels to his/her individual comfort level. In this regard, no standardized requirements existed regarding individual electrical programming parameters (frequency, current strength, pulse width, number and orientation of the electrode pole). Data collected was stored in the Relief register. Details about implantation and stimulation are described by Ashkan et al. [3]. For the purpose of this study, the stimulation parameters that the patients were currently using for the preventive treatment of chronic migraines were maintained.

\section{Statistical Analysis}

The data were evaluated using SPSS 27. The data were summarized as arithmetic mean and standard deviation as well as median, minimum, and maximum. The differences in the frequency of eyelid closure with experimental stimulation and without experimental stimulation were examined for significant differences using the Wilcoxon signed-rank test. The significance level $\alpha$ was set to 0.05 .

\section{RESULTS}

\section{Subjects}

Eight patients with chronic migraine, according to the diagnostic criteria of the International Headache Society (ICHD-2), who were treated with ONS were recruited. Six of them were women and two were men. The mean age was $47.63 \pm 16.08$ years (minimum 25 years, maximum 70 years). The patients had been treated with ONS for $11 \pm 3.44$ months (minimum 8 months, maximum 19 months).

\section{Orbicularis Oculi Reflex Depending on Active or Inactive Occipital Nerve Stimulation}

The blink frequency per minute between baseline and experimental triggering of the orbicularis oculi reflex depending on active or inactive occipital nerve stimulation is shown in Table 1 and Figs. 2 and 3. The baseline blink frequency measured without occipital nerve stimulation (20.63 \pm 17.55 eyelid closures/minute) or with active occipital nerve stimulation $(22.63 \pm 15.96$ eyelid closures/minute) did not differ significantly.

When the orbicularis oculi reflex was activated experimentally with standardized air flow directed to the cornea, the blink frequency increased to $39.38 \pm 21.85$ eyelid closures/ minute without occipital nerve stimulation. In contrast, active occipital nerve stimulation only showed an increase in the blink frequency to $30.00 \pm 17.22$ eyelid closures/minute (Fig. 2; $p=0.035)$.

Taking into account the difference to the respective baseline, the orbicularis oculi reflex $(7.38 \pm 20.14$ eyelid closures/minute) was significantly reduced on active ONS by $60.64 \%$ compared to inactive ONS $(18.75 \pm 14.30$ eyelid closures/minute) (Fig. 3; $p=0.021$ ). 
Table 1 Blink frequency per minute between baseline and experimental triggering of the orbicularis oculi reflex depending on active or inactive occipital nerve stimulation

\begin{tabular}{|c|c|c|c|c|c|}
\hline & & $\begin{array}{l}\text { ONS ON eyelid closures/ } \\
\text { min }\end{array}$ & $\begin{array}{l}\text { ONS OFF eyelid closures/ } \\
\text { min }\end{array}$ & Difference & $p$ value \\
\hline \multirow[t]{2}{*}{ Baseline } & Mean \pm SD & $22.63 \pm 15.96$ & $20.63 \pm 17.55$ & $2.00 \pm 13.94$ & 0.327 \\
\hline & $\begin{array}{l}\text { Median (min; } \\
\max )\end{array}$ & $20.00(8 ; 57)$ & $14.50(3 ; 55)$ & & \\
\hline \multirow{2}{*}{$\begin{array}{l}\text { With } \\
\text { airflow }\end{array}$} & Mean $\pm S D$ & $30.00 \pm 17.22$ & $39.38 \pm 21.85$ & $-9.38 \pm 10.84$ & 0.035 \\
\hline & $\begin{array}{l}\text { Median }(\min ; \\
\max )\end{array}$ & $26.50(13 ; 58)$ & $38.50(12 ; 84)$ & & \\
\hline \multirow[t]{2}{*}{ Difference } & Mean \pm SD & $7.38 \pm 20.14$ & $18.75 \pm 14.30$ & $-11.38 \pm 9.81$ & 0.021 \\
\hline & $\begin{array}{l}\text { Median (min; } \\
\max )\end{array}$ & $3.00(-20 ; 32)$ & $17.50(2 ; 42)$ & & \\
\hline
\end{tabular}

The arithmetic mean and standard deviation as well as median, minimum, and maximum values are given ( $p$ values in the Wilcoxon signed-rank test)

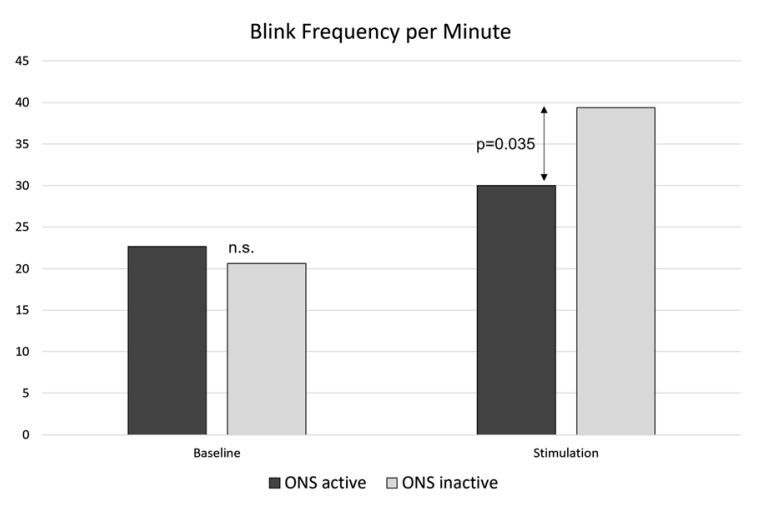

Fig. 2 Blink frequency per minute between baseline and experimental triggering of the orbicularis oculi reflex depending on active or inactive occipital nerve stimulation (Wilcoxon signed-rank test)

\section{DISCUSSION}

The study results show that with active ONS compared to inactive ONS in patients with chronic migraine, the orbicularis oculi reflex, dynamically triggered by a standardized air flow, is significantly reduced. This indicates that ONS is able to act directly on the trigeminally mediated central nociceptive stimulus processing in chronic migraine. The effects of aversive irritation are reduced. The effect of trigger

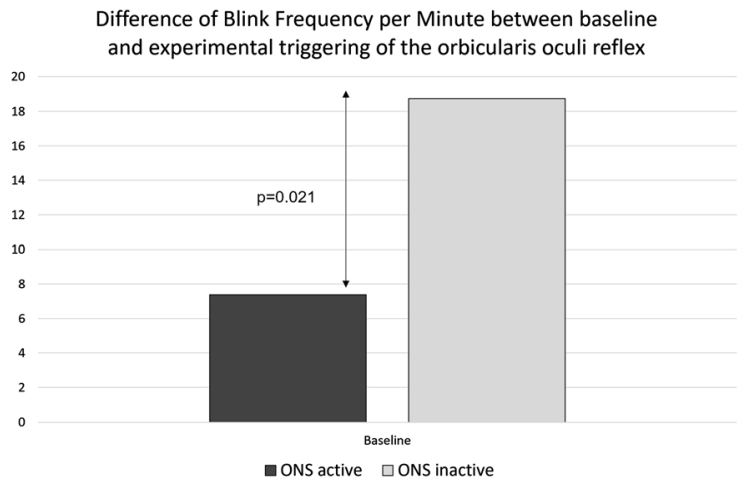

Fig. 3 Difference of blink frequency per minute between baseline and experimental triggering of the orbicularis oculi reflex depending on active or inactive occipital nerve stimulation (Wilcoxon signed-rank test)

factors in migraines could thus be weakened and the sensitization in the central nervous system reduced.

During the baseline measurement with or without active ONS, there was no aversive stimulation by standardized air flow. There was no difference between the ON and OFF states during the baseline phase. According to these results, ONS only seems to have an effect when an aversive stimulation occurs by standardized air flow leading to a nocifensive response. 
The results support the assumption that ONS has a therapeutic effect on the increased sensitization of pain circuitry with increased ventral posteromedial nucleus (VPM) neuronal firing [33]. As shown by Wodehouse et al. (2020), ONS can possibly alter the nociceptive traffic to the trigeminocervical complex, brainstem, and supranuclear connections [36]. These afferent pathways are modulated by the conditioned pain modulation (CPM) pathway, which is localized to the periaqueductal gray matter, nucleus raphe magnus, and the rostroventral medulla. An increased afferent input to the trigeminocervical complex (TCC) with increased peripheral sensitization and a generalized central sensitization of the trigeminospinal or the second order trigeminal neurons can contribute to the development of chronic headache. In addition, a disruption of the descending pain inhibitory pathway can lead to increased sensitization and be relevant in the chronification of headache [1, 2, 4, 17, 33, 36]. Increased activity in the descending pain pathway in ONS was also shown in a positron emission tomography (PET) study [1].

Our results support these findings. ONS leads to a significant reduction in the reflex response to trigeminally mediated aversive irritation. Interestingly, this appears to be an effect that requires active stimulation. After deactivating stimulation for $1 \mathrm{~h}$, a significant increase in the reflex response can already be seen. This indicates that continuous stimulation is required for achieving therapeutic goals. This is also supported by the fact that ONS is usually only clinically effective after several weeks of active stimulation and that the effect subsides briefly after stimulation is turned off or the battery is low [19]. The results are a further indication that ONS has a direct therapeutic effect on the trigeminocervical complex (TCC) and counteracts central sensitization.

The number of participants (eight) is a limitation of our study. On the one hand, patients with chronic migraines who are treated with ONS are a small group. On the other hand, they allow direct insight into trigeminal mechanisms by turning the stimulation on and off. In order to control the interindividual variability, we chose a balanced within-subject design.
Detailed comparative studies on the newly developed method, in particular on the electrically triggered blink reflex, are not yet available. The primary aim of the study was to investigate the mode of action of ONS on nocifensive mechanisms and pain-defending protective reflexes in patients. We have therefore developed and selected this new dynamic method with stimulation through a continuous standardized air flow in order to analyze its degree of selectivity regarding the therapeutic effects in this patient group. Regarding these results and a sensitive differentiation of the treatment effect, further investigations are to follow. Healthy controls were not added in this study, as the treatment effects can be compared directly in the crossover design (on-off). The experimental approach chosen aims to avoid pain and damage and includes a nocifensive mechanism, a pain defense reflex. The stimulation by the air flow on the cornea is aversive, which is why the reflex is triggered. Exactly which peripheral nerve fibers (tactile or nociceptive) are involved must remain open at the moment.

\section{CONCLUSIONS}

The results show that under-active ONS compared to inactive ONS in patients with chronic migraine, the orbicularis oculi reflex, dynamically physiologically triggered by a standardized air flow, is significantly reduced. This suggests that ONS is able to directly counteract the trigeminally mediated central sensitization in chronic migraine and protectively reduce the effects of aversive peripheral stimulation.

\section{ACKNOWLEDGEMENTS}

We thank the participants of the study.

Funding. No funding or sponsorship was received for this study or publication of this article.

Authorship. All named authors meet the International Committee of Medical Journal Editors (ICMJE) criteria for authorship for this 
article, take responsibility for the integrity of the work as a whole, and have given their approval for this version to be published.

Author contributions. Dr. Carl Göbel contributed to the design and conceptualization of the study, interpretation of the results, contributed to statistical analysis, and writing of the first draft of the manuscript. Dr. Axel Heinze contributed to interpretation and analysis. Sarah Karstedt contributed to interpretation and analysis. Dr. Svenja Clasen contributed to interpretation and analysis. Prof. Dr. Hartmut Göbel contributed to design and conceptualization of the study and co-writing of the manuscript.

Disclosures. Carl H. Göbel, Axel Heinze, Sarah Karstedt, Svenja Clasen and Hartmut Göbel declare they have nothing to disclose.

Compliance with Ethics Guidelines. The ethics committee of the medical faculty of the Christian-Albrechts University Kiel approved the study (D426/14). All subjects gave their informed written consent prior to participation. The study was performed in agreement with the Declaration of Helsinki. Written informed consent was obtained from all participants in this study for participation and publication.

Data Availability. The datasets generated during and/or analyzed during the current study are available from the corresponding author on reasonable request.

Open Access. This article is licensed under a Creative Commons Attribution-NonCommercial 4.0 International License, which permits any non-commercial use, sharing, adaptation, distribution and reproduction in any medium or format, as long as you give appropriate credit to the original author(s) and the source, provide a link to the Creative Commons licence, and indicate if changes were made. The images or other third party material in this article are included in the article's Creative Commons licence, unless indicated otherwise in a credit line to the material. If material is not included in the article's Creative Commons licence and your intended use is not permitted by statutory regulation or exceeds the permitted use, you will need to obtain permission directly from the copyright holder. To view a copy of this licence, visit http://creativecommons.org/licenses/by$\mathrm{nc} / 4.0 /$.

\section{REFERENCES}

1. Ahmed S, Plazier M, Ost J, et al. The effect of occipital nerve field stimulation on the descending pain pathway in patients with fibromyalgia: a water PET and EEG imaging study. BMC Neurol. 2018;18: 191.

2. Aibar-Duran JA, Alvarez Holzapfel MJ, Rodriguez Rodriguez $\mathrm{R}$, et al. Occipital nerve stimulation and deep brain stimulation for refractory cluster headache: a prospective analysis of efficacy over time. J Neurosurg. 2020. https://doi.org/10.3171/2019. 11.JNS192042.

3. Ashkan K, Sokratous G, Göbel H, et al. Peripheral nerve stimulation registry for intractable migraine headache (RELIEF): a real-life perspective on the utility of occipital nerve stimulation for chronic migraine. Acta Neurochir (Wien). 2020;162: 3201-11.

4. Boyer N, Dallel R, Artola A, et al. General trigeminospinal central sensitization and impaired descending pain inhibitory controls contribute to migraine progression. Pain. 2014;155:1196-205.

5. Chen YF, Bramley G, Unwin G, et al. Occipital nerve stimulation for chronic migraine-a systematic review and meta-analysis. PLoS ONE. 2015;10: e0116786.

6. Cruccu G, Ferracuti S, Leardi MG, et al. Nociceptive quality of the orbicularis oculi reflexes as evaluated by distinct opiate- and benzodiazepine-induced changes in man. Brain Res. 1991;556:209-17.

7. Dengler R. Orbicularis oculi reflex in clinical and experimental neurology. Fortschr Med. 1985;103: 556-8.

8. Dodick DW, Silberstein SD, Reed KL, et al. Safety and efficacy of peripheral nerve stimulation of the occipital nerves for the management of chronic migraine: long-term results from a randomized, multicenter, double-blinded, controlled study. Cephalalgia. 2015;35:344-58.

9. Garcia-Magro N, Negredo P, Martin YB, et al. Modulation of mechanosensory vibrissal responses 
in the trigeminocervical complex by stimulation of the greater occipital nerve in a rat model of trigeminal neuropathic pain. J Headache Pain. 2020;21:96.

10. Garcia-Ortega R, Edwards T, Moir L, et al. Burst occipital nerve stimulation for chronic migraine and chronic cluster headache. Neuromodulation. 2019;22:638-44.

11. Göbel A, Göbel $\mathrm{CH}$, Heinze A, et al. Imaging sensory effects of occipital nerve stimulation: a new computer-based method in neuromodulation. Brain Stimul. 2015;8:295-8.

12. Göbel CH, Göbel A, Niederberger U, et al. Occipital nerve stimulation in chronic migraine: the relationship between perceived sensory quality, perceived sensory location, and clinical efficacy-a prospective, observational, non-interventional study. Pain Ther. 2020;9:615-26.

13. Granot M. Can we predict persistent postoperative pain by testing preoperative experimental pain? Curr Opin Anaesthesiol. 2009;22:425-30.

14. Hacke W, Schaff C, Zeumer H. Orbicularis oculi reflex in computerized tomography verified lesions of the posterior cranial fossa. Fortschr Neurol Psychiatr. 1983;51:313-24.

15. Hann S, Sharan A. Dual occipital and supraorbital nerve stimulation for chronic migraine: a singlecenter experience, review of literature, and surgical considerations. Neurosurg Focus. 2013;35:E9.

16. Jenkins B, Tepper SJ. Neurostimulation for primary headache disorders, part 1: pathophysiology and anatomy, history of neuromodulation in headache treatment, and review of peripheral neuromodulation in primary headaches. Headache. 2011;51: 1254-66.

17. Kitaj MB, Klink M. Pain thresholds in daily transformed migraine versus episodic migraine headache patients. Headache. 2005;45:992-8.

18. Leon-Ariza JS, Prada DG, Leon-Ariza DS, et al. The three nociceptive responses of the orbicularis oculi reflex in Alzheimer's disease: state of the evidence and meta-analysis. Clin EEG Neurosci. 2019;50: 354-60.

19. Magis D, Gerardy PY, Remacle JM, et al. Sustained effectiveness of occipital nerve stimulation in drugresistant chronic cluster headache. Headache. 2011;51:1191-201.

20. Magown P, Garcia R, Beauprie I, et al. Occipital nerve stimulation for intractable occipital neuralgia: an open surgical technique. Clin Neurosurg. 2009;56:119-24.
21. Malin JP. The human orbicularis oculi reflex Some principal remarks. Electromyogr Clin Neurophysiol. 1982;22:45-53.

22. Miller S, Watkins L, Matharu M. Long-term outcomes of occipital nerve stimulation for chronic migraine: a cohort of 53 patients. J Headache Pain. 2016;17:68.

23. Picaza JA, Hunter SE, Cannon BW. Pain suppression by peripheral nerve stimulation. Chronic effects of implanted devices. Appl Neurophysiol. 1977;40: 223-34.

24. Rodrigo D, Acin P, Bermejo P. Occipital nerve stimulation for refractory chronic migraine: results of a long-term prospective study. Pain Physician. 2017;20:E151-9.

25. Saper JR, Dodick DW, Silberstein SD, et al. Occipital nerve stimulation for the treatment of intractable chronic migraine headache: ONSTIM feasibility study. Cephalalgia. 2011;31:271-85.

26. Schwedt TJ, Dodick DW, Trentman TL, et al. Occipital nerve stimulation for chronic cluster headache and hemicrania continua: pain relief and persistence of autonomic features. Cephalalgia. 2006;26:1025-7.

27. Serra G, Marchioretto F. Occipital nerve stimulation for chronic migraine: a randomized trial. Pain Physician. 2012;15:245-53.

28. Shimpo T. The orbicularis oris reflex and the orbicularis oculi reflex. Rinsho Shinkeigaku. $1985 ; 25: 271-81$.

29. Silberstein SD, Dodick DW, Saper J, et al. Safety and efficacy of peripheral nerve stimulation of the occipital nerves for the management of chronic migraine: results from a randomized, multicenter, double-blinded, controlled study. Cephalalgia. 2012;32:1165-79.

30. Silverstein LD, Graham FK, Calloway JM. Preconditioning and excitability of the human orbicularis oculi reflex as a function of state. Electroencephalogr Clin Neurophysiol. 1980;48:406-17.

31. Slotty PJ, Bara G, Kowatz L, et al. Occipital nerve stimulation for chronic migraine: a randomized trial on subthreshold stimulation. Cephalalgia. 2015;35:73-8.

32. Vernea J, Horvath T. Electromyographic studies of the orbicularis oculi reflex. Proc Aust Assoc Neurol. 1973;10:117-22.

33. Walling I, Smith H, Gee LE, et al. Occipital nerve stimulation attenuates neuronal firing response to mechanical stimuli in the ventral posteromedial 
thalamus of a rodent model of chronic migraine. Neurosurgery. 2017;81:696-701.

34. Weiner RL, Reed KL. Peripheral neurostimulation for control of intractable occipital neuralgia. Neuromodulation. 1999;2:217-21.

35. Wilbrink LA, Teernstra OP, Haan J, et al. Occipital nerve stimulation in medically intractable, chronic cluster headache. The ICON study: rationale and protocol of a randomised trial. Cephalalgia. 2013;33:1238-47.

36. Wodehouse T, Bahra A, Mehta V. Changes in peripheral and central sensitization in patients undergoing occipital nerve stimulation. Br J Pain. 2020;14:250-5.

37. Young WB. Occipital nerve stimulation for chronic migraine. Curr Pain Headache Rep. 2014;18:396. 